\title{
MAN.05 - Therapeutic horizon for acute limphoblastic leukemia: a technology foresight study
}

Hugo Garcia Tonioli Defendi ${ }^{1 *}$; Fabíola Araujo Cordeiro ${ }^{1}$; Tatiana Jorge Fernandes ${ }^{1}$; Aline de Almeida Oliveira ${ }^{1}$.

1Fiocruz/Bio-Manguinhos.

Introduction: Acute Lymphoblastic Leukemia (ALL) is a type of cancer of great relevance in public health, being considered the most frequent in childhood. Currently, standard therapy is considered effective, but many new technologies have reached clinical trials, what can change the treatment of ALL in the future. The methods of technological foresight, as tech mining and expert opinion, are widely used by companies in order to minimize the risks of investments and to understand the opportunities and threats of a certain field. In this context, fostering a broad and future vision, this work aimed the identification of potential technologies that could benefit patients with ALL to be invested by Bio-Manguinhos.

Objective: Perform exploratory analysis of the global scenario of developed and under development medicines for the ALL treatment, through a combination of technology foresight methods, with the aim to understand the better strategy to be pursued by Bio-Manguinhos.

Methodology: Firstly, a search in the Medtrack database was applied in order to select products indicated to ALL. These set of data were exported to Excel10 and then a classification in terms of technology and clinical strategies was done for each project/product identified. Frequency graphs were generated and correlations between technology, clinical and status of development were done. After, a pre-validation process was undertook with physicians.

Results: The Medtrack database returned 146 results, including 71 products under development, 53 small molecules, 17 biological reference and 5 biosimilars. The focus of the study was to analyse the innovative products with potential to be launched in the market. Within the innovative products, we identified 33 small molecules, 31 biologicals and 4 semi-synthetics. The biotechnological products were sub-classified in cellular therapy (16), monoclonal antibody (13) and enzymes (6). The data shows a Pharmaceutical repositioning in terms of investment, highlighting the cellular therapy with most of the products in clinical phase of development. Monoclonal antibodies also represent a relevant shift. On the other hand, the enzymes, most used in the current clinical practice appeared in the last position. In what concerns to the innovative small molecules, the anti-metabolic agents (9) and protein kinase inhibitor (9) appeared as the most important technologies. The clinical analysis and pre-validation step showed that enzymes, although not the main target of investment by pharmaceutical industries, remain as a good investment option for Bio-Manguinhos in the recombinant and/or pegylated forms, because its properties in the clinical practice.

Conclusion: The combination of tech mining and pre-validation with expert opinion methods provided an assertive overview of the current and future scenario of medicines to treat ALL. The conclusion until now, that will be validated in an experts panel, is that l-asparaginase in a recombinant and/or pegylated form seems to be a promise product to be invested by Bio-Manguinhos.

Keywords: Technology Foresight; Acute Lymphoblastic Leukemia; L-asparaginase 